\title{
Evaporation from weighing precipitation gauges: impacts on automated gauge measurements and quality assurance methods
}

\author{
R. D. Leeper ${ }^{1}$ and J. Kochendorfer ${ }^{2}$ \\ ${ }^{1}$ Cooperative Institute for Climate and Satellites (CICS) North Carolina State University and NOAA's \\ National Climatic Data Center (NCDC), 151 Patton Ave., Asheville, NC 28801, USA \\ ${ }^{2}$ NOAA's Atmospheric Turbulence Diffusion Division (ATDD), 456 S. Illinois Ave., Oak Ridge, TN 37830, USA \\ Correspondence to: R. D. Leeper (ronald.leeper@noaa.gov)
}

Received: 31 October 2014 - Published in Atmos. Meas. Tech. Discuss.: 20 December 2014

Revised: 2 April 2015 - Accepted: 28 April 2015 - Published: 4 June 2015

\begin{abstract}
Evaporation from a precipitation gauge can cause errors in the amount of measured precipitation. For automated weighing-bucket gauges, the World Meteorological Organization (WMO) suggests the use of evaporative suppressants and frequent observations to limit these biases. However, the use of evaporation suppressants is not always feasible due to environmental hazards and the added cost of maintenance, transport, and disposal of the gauge additive. In addition, research has suggested that evaporation prior to precipitation may affect precipitation measurements from autorecording gauges operating at sub-hourly frequencies. For further evaluation, a field campaign was conducted to monitor evaporation and its impacts on the quality of precipitation measurements from gauges used at U.S. Climate Reference Network (USCRN) stations. Two Geonor gauges were collocated, with one gauge using an evaporative suppressant (referred to as Geonor-NonEvap) and the other with no suppressant (referred to as Geonor-Evap) to evaluate evaporative losses and evaporation biases on precipitation measurements. From June to August, evaporative losses from the GeonorEvap gauge exceeded accumulated precipitation, with an average loss of $0.12 \mathrm{~mm} \mathrm{~h}^{-1}$. The impact of evaporation on precipitation measurements was sensitive to the choice of calculation method. In general, the pairwise method that utilized a longer time series to smooth out sensor noise was more sensitive to gauge evaporation $(-4.6 \%$ bias with respect to control) than the weighted-average method that calculated depth change over a smaller window $(<+1 \%$ bias $)$. These results indicate that while climate and gauge design affect gauge evaporation rates, computational methods also influence the magnitude of evaporation biases on precipitation
\end{abstract}

measurements. This study can be used to advance quality insurance (QA) techniques used in other automated networks to mitigate the impact of evaporation biases on precipitation measurements.

\section{Introduction}

In situ observations of precipitation are an integral component of hydrological studies (drought, flooding, etc.), and are often used to correct and validate radar, satellite, and modeled estimates of precipitation (Wang and Wolff, 2010). However, point measurements of precipitation have well known biases (Sevruk and Hamon, 1984; Goodison et al., 1981; Lanza et al., 2005; and Sieck et al., 2007) (e.g., wind, gauge evaporation, wetting factor, and observer errors) that impact the quality of precipitation measurements. International studies organized by the World Meteorological Organization (WMO) have fostered partnerships and collaborations since the 1990s focused on these sources of error in an effort to improve precipitation measurements (Sevruk et al., 2009). A majority of these investigations have concentrated on wind-induced undercatch and the development of methods to correct for such errors (Sevruk et al., 2009). However, for automated networks with well-shielded precipitation gauges, such as the U.S. Climate Reference Network (USCRN), gauge evaporation is also an important source of observational error to consider.

Yang et al. (1998) defines gauge evaporation errors as the amount of precipitation loss prior to observation, which suggests that the effects of gauge evaporation can be mitigated 
Table 1. The set of quality control checks both QA methods apply during the evaluation of depth change.

\begin{tabular}{ll}
\hline Check description & Constraint \\
\hline Gauge capacity (max. gauge depth) & $600 \mathrm{~mm}$ (most gauges) \\
Sensor health (min. gauge depth) & Less than $-10 \mathrm{~mm}$ \\
Gauge maintenance (max. depth change) & $25 \mathrm{~mm} 5 \mathrm{~min}^{-1}$ \\
Precipitation threshold (min. depth change) & $0.2 \mathrm{~mm} 5 \mathrm{~min}^{-1}$ \\
\hline
\end{tabular}

with frequent observations as noted in WMO (2008). The magnitude of evaporative loss from precipitation gauges vary by season (Dunne and Leopold, 1978; Aaltonen et al., 1993; Strangeways, 2004) and are more pronounced in warm-dry climates (WMO, 2008), due to greater evaporative demand. Gauge design has also been found to affect evaporation rates (Sevruk, 1974; Golubev et al., 1992). Gauges with an open (no-funnel) reservoir had higher evaporation rates as the internal moisture (internal reservoir and walls) is more exposed to the atmosphere. Golubev et al. (1992) noted the automated non-funnel capped Tretyakov gauge had evaporation rates $\left(1.15 \mathrm{~mm} \mathrm{day}^{-1}\right) 6$ times greater than the manual funnel-capped standard $8^{\prime \prime}$ gauge $\left(0.19 \mathrm{~mm}\right.$ day $\left.^{-1}\right)$ used at National Weather Service (NWS) Cooperative Observer Program (COOP) stations. Precipitation biases due to gauge evaporation can be up to $4 \%$ (WMO, 2008), which is nearly equivalent to the $5 \%$ annual average change in precipitation reported in the most recent National assessment (Walsh et al., 2014). To improve the quality of precipitation measurements, the WMO (2008) suggest taking observations frequently to reduce the magnitude of evaporation per observation cycle and making use of evaporative suppressants.

However, recent research comparing USCRN with COOP stations indicate gauge evaporation can bias observations even when taken frequently at a sub-hourly rate (Leeper et al., 2015a). The USCRN monitors precipitation at a $5 \mathrm{~min}$ frequency from a well-shielded automated funnel-less gauge whereas COOP stations operate a manual funnel-capped gauge that is checked daily. Despite COOP stations monitoring precipitation from an unshielded gauge, USCRN observations of precipitation (daily aggregated sum of sub-hourly data) were slightly less than COOP by $1.5 \%$ (Leeper et al., 2015a). Network differences were not as large for northernlocated station pairs that had a greater percentage of frozen hydrometeors, which are more sensitive to surface wind and where gauge shielding has a more dominant impact on catch efficiency (Leeper et al., 2015a). Overall, these results are contrary to other studies that found unshielded gauges tended to report 4 to $11 \%$ less precipitation for liquid hydrometeors (Golubev et al., 1992; and Duchon and Essenberg, 2001), although neither included the Geonor gauge used at USCRN stations. A portion of the dry bias between USCRN and COOP, among others (i.e., wetting factor, observer error), was thought to be attributed to computational methods within the quality assurance (QA) system that may be sensitive to sensor noise and gauge evaporation (Leeper et al., 2015a). Further analysis of the QA system, using synthetic precipitation events of a known precipitation signal revealed the method used to calculate depth change was sensitive to simulated gauge evaporation and sensor noise signals (Leeper et al., 2015b). In these cases, evaporative decreases in gauge depth occurring immediately prior to a precipitation event lead to overestimates of the initial gauge depth, which resulted in an underestimate of total depth change (current minus initial gauge depths) or precipitation (Leeper et al., $2015 b)$. These results ultimately lead to the development of a new QA system that was less sensitive to sensor noise and gauge evaporation in the same synthetic precipitation event tests (Leeper et al., 2015b). These studies suggest that while evaporative demand and precipitation gauge design influence gauge evaporation rates, the techniques used in QA methods to evaluate depth change (quantify precipitation) also impact the magnitude of gauge evaporation bias on precipitation measurements.

To evaluate the impact of gauge evaporation on QA processes, a field experiment was conducted over the summer of 2013 with two Geonor T-200B all-weather precipitation gauges equipped with redundant load sensors and initialized with and without an evaporative suppressant. The purpose of this study is to quantify evaporative losses from the Geonor gauge used at USCRN stations and evaluate the effectiveness of calculation methods to monitor precipitation at sub-hourly frequency without the use of evaporative suppressants. To the authors' knowledge, this is the first study to quantify evaporative losses from the Geonor T-200B gauge. Given that the impacts of gauge evaporation on precipitation measurements extends to other networks beyond the USCRN, this study may provide valuable insights to the development of QA methods that are less sensitive to gauge evaporative biases. This is particularly true for networks operating gauges with an exposed reservoir and an insufficient quantity of evaporative suppressant.

\section{Algorithm descriptions}

Since detailed descriptions of both methods and comparisons are provided in Leeper et al. (2015b), brief descriptions of the two QA methods are provided here as a reference. The two QA methods evaluated in this study were both designed for the same USCRN precipitation system. For this 


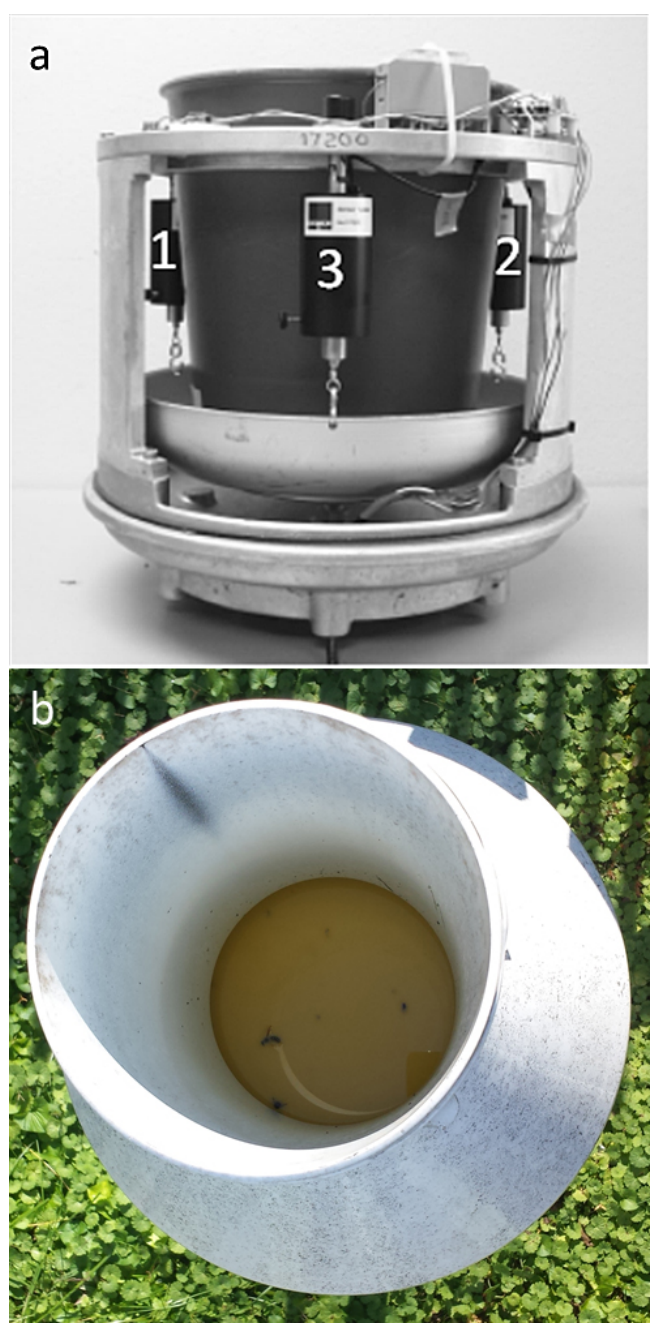

Figure 1. Photographs of the Geonor T200B gauge (a) interior outfitted with redundant sensors and (b) looking through the gauge opening to the exposed interior reservoir.

reason, both methods share the same QA checks noted in Baker et al. (2005) and identified in Table 1. These checks ensure the gauge is operating normally; within gauge capacity ( $600 \mathrm{~mm}$ for the gauges used in this study) and with functioning load sensors (failing sensors report negative gauge depths). To identify instances of gauge maintenance (which often includes sensor calibration), a maximum depth change of $25 \mathrm{~mm}$ per $5 \mathrm{~min}$ period is also applied. A minimum depth change (detection) limit of $0.2 \mathrm{~mm}$ is also used to reduce the occurrence of false precipitation estimates due to sensor noise (C. B. Baker, personal communication, 2015). However, the accuracy of the gauge above this minimum detection limit is $0.1 \mathrm{~mm}$ (i.e., QA methods can report increases in gauge depth of $0.3 \mathrm{~mm}$ ). In both algorithms, if any of the load sensors fail these checks it is not used to evaluate precipitation. Moreover, the primary difference between the two QA algorithms is how a single depth change is computed from the triplicate measurements from each gauge as described in the proceeding paragraphs.

The initial QA methodology computes depth change for each load sensor as the current depth minus a $2 \mathrm{~h}$ average of previous gauge depths (to smooth sensor noise), provided no precipitation has been observed in the preceding $2 \mathrm{~h}$. If precipitation has been observed, depth change is calculated as the difference between the current depth and the depth at the time of last reported precipitation. Once the individual depth changes (deltas) are computed for each of the three load sensors, they are compared to one another using a pairwise approach to determine which load sensors (also referred to as wires) will be used in the final calculation. This is similar to the approach USCRN uses to integrate redundant measures of atmospheric temperature. Only wire deltas that agree to within $0.2 \mathrm{~mm}$ pass this check and are used to calculate precipitation. The wires that pass the pairwise check are averaged together using a simple arithmetic mean. As a final check, if a collocated rain detector has detected precipitation for this period, the mean is rounded to the nearest 10 th decimal place and recorded as precipitation. This approach is referred to as the pairwise method throughout the remainder of this study with additional information provided in Leeper et al. (2015b).

A more recent approach was developed that computes depth change over the $5 \mathrm{~min}$ sub-hourly period (current depth minus most recent depth) for each load sensor. This is similar to the way a human observer might process the gauge data and is one of the fundamental differences between the two methods. Previous gauge data $(2 \mathrm{~h})$ is used in this approach to weight each wire by the inverse of its variance (a measure of sensor noise) from the three-wire delta mean. Precipitation is then calculated from the weighted average of the three load sensors and rounded to the nearest $0.1 \mathrm{~mm}$, provided precipitation has been detected by the collocated rain detector. This approach will be referred to as the wAvg method for the remainder of this study. Additional details regarding the function and performance of this approach is also available in Leeper et al. (2015b).

\section{Methodology}

To observe precipitation, the USCRN uses the all-weather Geonor T-200B gauge equipped with three redundant load sensors as shown in Fig. 1a. Each of the load sensors has an internal wire that when plucked vibrates at a frequency that changes with the tension on the wire as described by Duchon (2008). When calibrated, the resonant frequency of the wire is converted to gauge depth, which is used to monitor precipitation (Leeper et al., 2015b). The redundant observation system preserves the quality and the continuity of the data record by allowing QA processes to detect and remove degraded or failing sensors while reducing the frequency of data gaps as the additional sensors continue to 


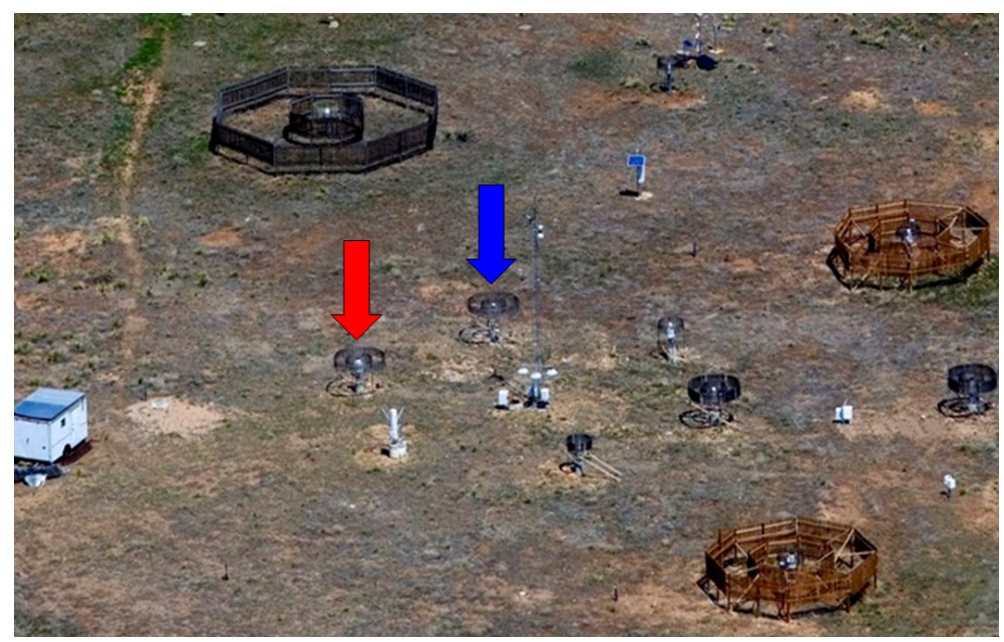

Figure 2. Image of Geonor-Evap (blue arrow) and Geonor-NonEvap (red arrow) gauges used in this study.

operate (Leeper et al., 2015b). In addition to supporting redundant monitoring, the Geonor gauge is also designed with an open vertical shaft (Fig. 1b) to limit splashing out (hydrometers splashing out of the gauge) and snow/ice capped (orifice) errors. The USCRN Geonor gauges also have inlet heaters installed on them to prevent orifice capping caused by snow and ice; however, these heaters are only operated at freezing temperatures and were never activated during this study.

Unfortunately, this design also exposes the internal reservoir to the atmosphere, resulting in gauge evaporation if a suppressant is not used. The USCRN does not use an evaporative suppressant in network gauges unless it is necessary for the operation of the gauge. For instance, most northern stations during winter will have a suppressant mixture to limit the evaporation of antifreeze used to melt frozen hydrometeors so the gauge can continue to report liquid equivalent precipitation totals. These suppressants are not added to all gauges, and when applied are only used over the winter season. Moreover, gauge evaporation biases are still a factor over summer months when evaporative demand is higher.

To quantify evaporation rates from the Geonor gauge and evaluate QA performance, a field experiment was performed over the 2013 summer (June to August) at the NOAA/FAA/NCAR Winter Precipitation Test Bed in Marshall, CO (described in Rasmussen et al., 2012). The campaign consisted of two identical Geonor T-200B gauges surrounded by double-Alter shields that were collocated within $10 \mathrm{~m}$ as shown in Fig. 2. The northern gauge (GeonorEvap) was setup without an evaporative suppressant with an initial gauge depth of $\sim 277 \mathrm{~mm}$. The Geonor-Evap gauge was compared the control gauge Geonor-NonEvap, which had an evaporative suppressant mixture of antifreeze $(60 \%$ methanol and $40 \%$ glycol) and $300 \mathrm{~mL}$ of automatic transmission fluid added to limit gauge evaporation. Observations from redundant load sensors in both gauges were stored every minute. Using an independent rain detector (Vaisala DRD11A, Helsinki, Finland) to differentiate between wet and dry periods, changes in gauge depth from the paired gauges were used to quantify evaporative loss (during dry periods) and evaluate biases as a result of evaporation on precipitation measurements during rainy conditions. This rain detector was installed on a tower located $10 \mathrm{~m}$ from the two gauges at a height of $1.5 \mathrm{~m}$.

The most direct approach to quantify evaporative loss from the Geonor gauge is to compare depth changes between Geonor-Evap and Geonor-NonEvap gauges over dry periods. Changes in depth were evaluated hourly from $1 \mathrm{~min}$ observations by subtracting the last minute depth from the first, and then averaging over the redundant sensors in each gauge. To evaluate the sensitivity of gauge evaporation to atmospheric conditions, air temperature (Thermometrics $1000 \Omega$ Platinum Resistance Thermometer housed in a MetOne 0766B Fan Aspirated Radiation Shield), humidity (Vaisala HMT337), and wind speed (MetOne 014A Wind Speed Sensor) were also monitored throughout the study period from a height of $1.5 \mathrm{~m}$. The sensors that monitor these variables were located on separate towers near the study gauges with temperature, humidity, and wind speed measurements taken approximately 10,37 , and $67 \mathrm{~m}$ away respectively. In addition, a USB temperature logger (EL-USB-1) was submerged within the reservoir of the Geonor-Evap gauge to monitor the internal water temperature.

To evaluate QA performance, gauge data from the collocated Geonor-Evap and Geonor-NonEvap gauges were processed through two QA systems for precipitation and compared, resulting in a total of four series: Geonor-Evap_wAvg, Geonor-Evap_pairwise, GeonorNonEvap_wAvg, and Geonor-NonEvap_pairwise. To conform to the USCRN $5 \mathrm{~min}$ temporal resolution that is required by the QA methods, the 1 min observations from the experimental gauges were subsampled to $5 \mathrm{~min}$ periods by 


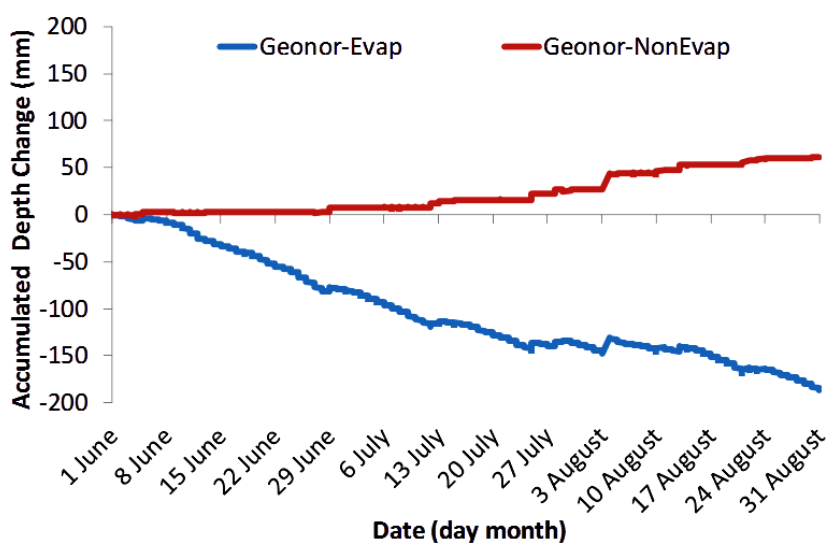

Figure 3. Hourly three-wire mean depth change over the 3-month study period in 2013 .

simply taking the 1 min observation corresponding to each 5 min period of the hour $(0,5,10,15, \ldots, 55)$. Precipitation comparisons between Geonor-Evap and the GeonorNonEvap (control) gauges using the same QA algorithm will reveal how sensitive each method is to gauge evaporation. In other words, QA reported precipitation should be similar between the two gauges if gauge evaporation is not a factor. Differences were analyzed over the 3-month study period and for each precipitation event, with events defined as a continuous period of precipitation with a $2 \mathrm{~h}$ period of no precipitation at the beginning and end of each event. While QA algorithm differences from the same gauge (Geonor-Evap or Geonor-NonEvap) are expected, this is not the focus of this study, which already has been extensively analyzed in Leeper et al. (2015b).

\section{Results}

\subsection{Study period conditions}

Over the 3-month study period, air temperature and total precipitation were typical of Boulder Colorado summers (COOP 050848; http://www.wrcc.dri.edu/cgi-bin/cliMAIN. pl?co0848) that have an average (1893-2013) temperature and precipitation total of $21.2^{\circ} \mathrm{C}$ and $131.3 \mathrm{~mm}$ respectively. Air temperature ranged from 34.3 to $5.7^{\circ} \mathrm{C}$ with a slightly warmer mean $\left(21.3^{\circ} \mathrm{C}\right)$ than the gauge evaporation study conducted in Valdai Russia by Golubev et al. (1992). The atmosphere was also drier with mean relative humidity and dew point temperatures of $44.6 \%$ and $7.6^{\circ} \mathrm{C}$ respectively. These conditions were ideal for a gauge evaporation field study, which was reflected in large vapor pressure deficits of $2.6 \mathrm{kPa}$ on average. Vapor pressure deficit, the difference between vapor pressure of saturated air and current atmosphere conditions, is directly proportional to evaporation rates (Dunne and Leopold, 1978). From June to August, there were a total of 29 precipitation events (continuous precipita-

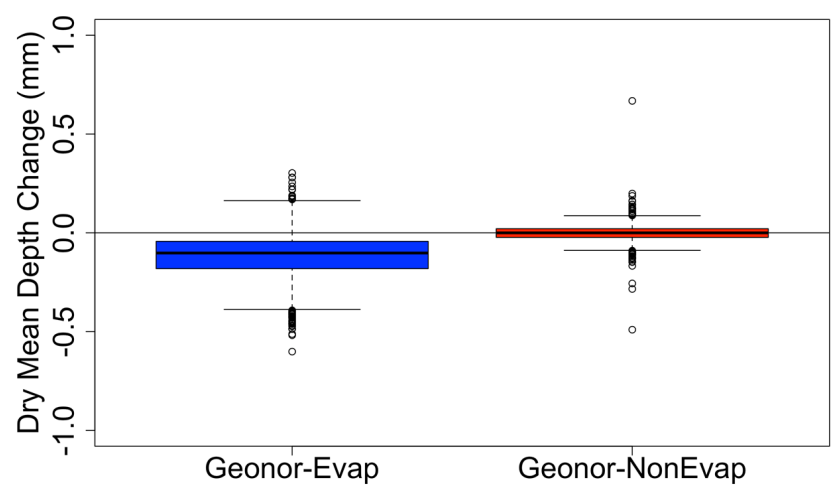

Figure 4. Box plots of the three-load sensor hourly mean depth change over the dry period from Geonor-Evap $(\sigma=0.12)$ and Geonor-NonEvap $(\sigma=0.04)$ gauges.

tion with 2 or more hours of no precipitation between events), totaling $244 \mathrm{~h}$ of atmospheric wetness as observed from the nearby rain detector. The results include two subsections: dry conditions to quantify evaporative loss from Geonor gauges, and wet conditions to evaluate the impact of gauge evaporation on reported precipitation.

\subsection{Dry conditions}

Comparisons of depth change between gauges clearly reveal an evaporation signal (Fig. 3). Over the 3-month study period, evaporative losses from the Geonor-Evap gauge totaled $228.5 \mathrm{~mm}$ over dry non-rainy hours. Similar losses in gauge depth were not reported from the Geonor-NonEvap gauge $(2.6 \mathrm{~mm})$. Average hourly losses from Geonor-Evap and Geonor-NonEvap gauges over the dry period were 0.1 and $0.0 \mathrm{~mm} \mathrm{~h}^{-1}$ respectively. The Geonor-NonEvap gauge had a smaller distribution of hourly depth change over the dry period that was more symmetric (about zero) than the GeonorEvap gauge (Fig. 4), which over the study period averaged to zero. Variations in depth from the Geonor-NonEvap gauge are likely the result of sensor noise caused by temperature and wind speed variations described by Duchon (2008). In addition, the Geonor-NonEvap gauge has a smaller root mean squared error (RMSE) of $0.04 \mathrm{~mm}$ than the GeonorEvap gauge $(0.16 \mathrm{~mm})$, assuming any change in gauge depth over dry periods is considered an error. The mean reductions in depth from the Geonor-NonEvap gauge were of similar magnitude to the NWS standard $8^{\prime \prime}$ gauge reported in the Golubev et al. (1992) study. The larger variations in depth change from the Geonor-Evap gauge suggest that the gauge evaporation signal is larger than sensor noise and causes a negative bias in depth change. Depth changes over the dry period from the Geonor-NonEvap gauge were considered negligible with average losses, and RMSE values were less than the accuracy of the instrument $(0.1 \mathrm{~mm})$.

Evaporative losses from the Geonor-Evap gauge were well correlated with atmospheric conditions conducive to 

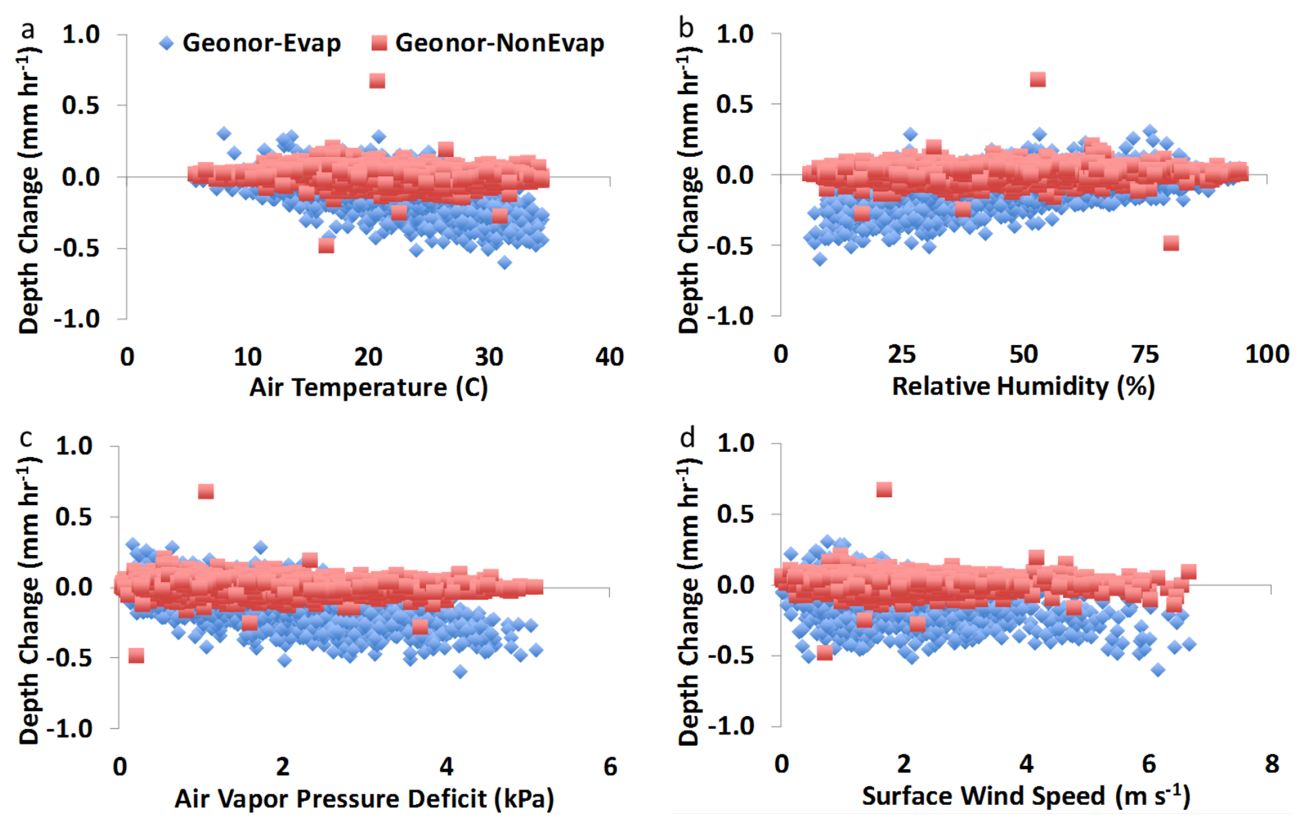

Figure 5. Hourly three-wire mean depth change over dry periods for Geonor-Evap and Geonor-NonEvap gauges by (a) air temperature, (b) relative humidity, (c) air-vapor pressure deficit, and (d) surface wind speed.
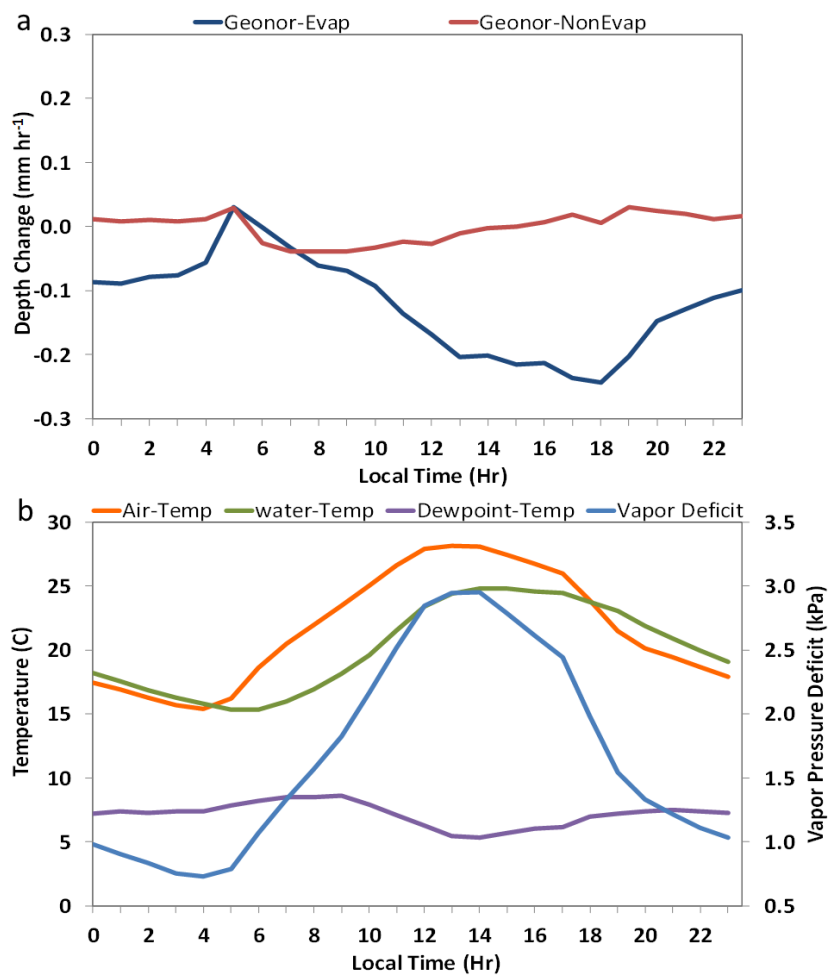

Figure 6. Hourly mean (a) three-wire depth change over dry periods for Geonor-Evap and Geonor-NonEvap gauges and (b) air and water temperatures and air-vapor pressure deficit over the diurnal cycle. evaporation. Hourly depth changes from the Geonor-Evap gauge were generally more negative during warm (Fig. 5a) and dry (Fig. 5b and c) conditions when evaporative demand was highest. However, surface winds seemed to have a smaller impact on gauge evaporation, with large reductions $\left(-0.5 \mathrm{~mm} \mathrm{~h}^{-1}\right)$ in gauge depth observed regardless of wind speed (Fig. 5d). The lack of an apparent relationship with wind speed may be due to the fact that the volume of air within the gauge is well shielded from the wind and that convection caused by solar heating of the gauge dominates the exchange rather than wind-driven mixing in this setup. Hourly increases in gauge depth at lower winds speeds (between 0 and $2 \mathrm{~m} \mathrm{~s}^{-1}$ ), which reduced with increasing wind speed, may be related to the lack of an evaporation signal where gauge depth changes would be driven by sensor noise (slight positive and negative changes as shown from Geonor-NonEvap) and or condensation buildup on the gauge reservoir during the early morning hours when surface winds were generally calm. However, this was not fully explored in this study. For the Geonor-NonEvap gauge, changes in gauge depth were generally smaller than Geonor-NonEvap and symmetric about zero regardless of atmospheric conditions.

Similarly, diurnal variations in gauge depth were larger for the Geonor-Evap than Geonor-NonEvap gauge (Fig. 6a). Over the diurnal cycle, Geonor-Evap depth change was mostly negative, with the largest average reduction of $0.2 \mathrm{~mm} \mathrm{~h}^{-1}$ at 18:00 LT when reservoir (water) temperatures were warmest (Fig. 6b). Over evening hours, mean hourly losses from Geonor-Evap gauge were moderated compared to daylight hours as the evaporation demand decreased. The 
Table 2. Reported precipitation event totals from the Geonor-Evap and Geonor-NonEvap gauges and gauge differences (Geonor-Evap Geonor-NonEvap) using pairwise and wAvg algorithms.

\begin{tabular}{|c|c|c|c|c|c|c|}
\hline \multirow[b]{2}{*}{ EventID } & \multicolumn{2}{|c|}{ Geonor-Evap } & \multicolumn{2}{|c|}{ Geonor-NonEvap } & \multicolumn{2}{|c|}{ Gauge Difference } \\
\hline & Pairwise (mm) & wAvg (mm) & Pairwise (mm) & wAvg (mm) & Pairwise (mm) & wAvg (mm) \\
\hline 1 & 2.2 & 2.3 & 2.1 & 2.0 & 0.1 & 0.3 \\
\hline 2 & 0.0 & 0.3 & 0.0 & 0.2 & 0.0 & 0.1 \\
\hline 3 & 0.0 & 0.0 & 0.3 & 0.3 & -0.3 & -0.3 \\
\hline 4 & 5.1 & 5.5 & 5.6 & 5.4 & -0.5 & 0.1 \\
\hline 5 & 3.3 & 3.7 & 3.7 & 3.7 & -0.4 & 0.0 \\
\hline 6 & 1.5 & 1.6 & 1.4 & 1.5 & 0.1 & 0.1 \\
\hline 7 & 0.6 & 0.6 & 0.6 & 0.6 & 0.0 & 0.0 \\
\hline 8 & 0.8 & 0.9 & 0.8 & 0.9 & 0.0 & 0.0 \\
\hline 9 & 0.3 & 0.2 & 0.0 & 0.3 & 0.3 & -0.1 \\
\hline 10 & 0.0 & 0.2 & 0.2 & 0.3 & -0.2 & -0.1 \\
\hline 11 & 0.0 & 0.0 & 0.2 & 0.2 & -0.2 & -0.2 \\
\hline 12 & 0.2 & 0.0 & 0.2 & 0.2 & 0.0 & -0.2 \\
\hline 13 & 6.7 & 7.0 & 6.6 & 6.8 & 0.1 & 0.2 \\
\hline 14 & 3.8 & 3.9 & 3.6 & 3.6 & 0.2 & 0.3 \\
\hline 15 & 0.7 & 0.8 & 0.5 & 0.7 & 0.2 & 0.1 \\
\hline 16 & 0.6 & 0.6 & 0.5 & 0.5 & 0.1 & 0.1 \\
\hline 17 & 0.3 & 0.5 & 0.3 & 0.3 & 0.0 & 0.2 \\
\hline 18 & 0.0 & 0.3 & 0.3 & 0.3 & -0.3 & 0.0 \\
\hline 19 & 16.7 & 17.2 & 16.9 & 16.8 & -0.2 & 0.4 \\
\hline 20 & 0.3 & 0.4 & 0.5 & 0.7 & -0.3 & -0.3 \\
\hline 21 & 3.5 & 3.7 & 3.4 & 3.6 & 0.1 & 0.1 \\
\hline 22 & 0.0 & 0.3 & 0.3 & 0.3 & -0.3 & 0.0 \\
\hline 23 & 5.2 & 5.3 & 5.2 & 5.2 & 0.0 & 0.1 \\
\hline 24 & 0.0 & 0.2 & 0.3 & 0.3 & -0.3 & -0.1 \\
\hline 25 & 4.4 & 4.7 & 4.7 & 4.8 & -0.3 & -0.1 \\
\hline 26 & 1.4 & 1.6 & 1.8 & 1.7 & -0.4 & -0.1 \\
\hline 27 & 0.0 & 0.3 & 0.6 & 0.4 & -0.6 & -0.1 \\
\hline 28 & 0.0 & 0.0 & 0.0 & 0.2 & 0.0 & -0.2 \\
\hline 29 & 0.0 & 0.2 & 0.0 & 0.3 & 0.0 & -0.1 \\
\hline Total & 57.6 & 62.3 & 60.6 & 62.1 & -3.0 & 0.2 \\
\hline
\end{tabular}

reduction in evaporative demand suggests that depth variations from the Geonor-Evap gauge may have been influenced more by sensor noise (similar to Geonor-NonEvap) as noted earlier, which may help to explain the small mean increase $(+0.03 \mathrm{~mm})$ in gauge depth at 05:00 LT. A diurnal signal was also detected from the Geonor-NonEvap gauge due to sensor noise, which had a much smaller amplitude and dissimilar periodicity (timing of peak and trough) compared to the Geonor-Evap gauge. The largest average decrease $\left(0.04 \mathrm{~mm} \mathrm{~h}^{-1}\right)$ over the diurnal scale from the control (Geonor-NonEvap) gauge was much smaller than changes reported from Geonor-Evap gauge. However, it is interesting to note that the Geonor-Evap and GeonorNonEvap gauges both experienced small positive depth changes $(\sim+0.03 \mathrm{~mm})$ at 05:00 LT. The coincident timing may indicate that on days when condensation buildup occurred (likely not every day) it was observed within the same hour for both gauges even though the mean depth change was well below the $0.1 \mathrm{~mm}$ accuracy of the gauge.

\subsection{Wet conditions}

The impact of gauge evaporation on calculated precipitation was discernable, but dependent on the QA method. Precipitation differences between Geonor-Evap and Geonor-NonEvap gauges were generally larger when using the pairwise algorithm (Fig. 7). Overall, the pairwise method reported $3.0 \mathrm{~mm}$ $(4.9 \%)$ more precipitation from the Geonor-NonEvap than Geonor-Evap gauge, with the Geonor-NonEvap gauge reporting $0.1 \mathrm{~mm}$ more precipitation per event (see Table 2). The wAvg algorithm reported $0.2 \mathrm{~mm}(-0.3 \%)$ less precipitation from the Geonor-NonEvap gauge for a negligible average event difference of $+0.01 \mathrm{~mm}$ event $^{-1}$. Time series of the accumulated precipitation difference between gauges (Geonor-Evap minus Geonor-NonEvap) reveal that the pairwise method consistently reported more precipitation from 


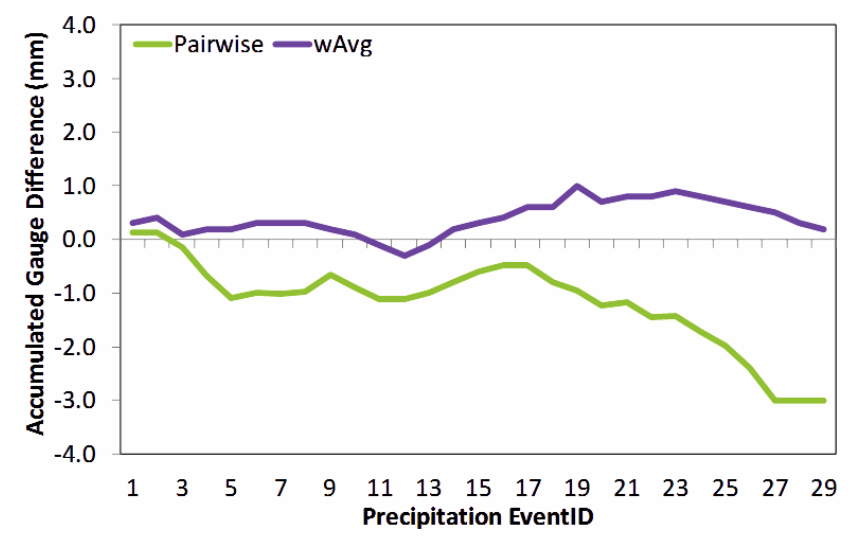

Figure 7. Accumulated precipitation differences between GeonorEvap and Geonor-NonEvap gauges (Geonor-Evap - GeonorNonEvap) computed from pairwise and wAvg calculation methods.

the Geonor-NonEvap gauge (Fig. 7). Conversely, wAvg differences were variable in sign, which was likely caused by natural variations in the spatial distribution of precipitation and other sources of gauge (sensor noise) error.

Examination of QA processes revealed that methods used to determine depth change within the algorithms impacted their sensitivity to gauge evaporation. To compute depth change, both QA methods difference the current gauge depth from a reference depth (currentDepth - referenceDepth). Pairwise, which calculates a reference depth at the beginning of an event as the average of previous depths $(2 \mathrm{~h})$, tended to report negative depth changes prior to precipitation events (Fig. 8a-f) from the Geonor-Evap gauge. In other words, the time-averaged $(2 \mathrm{~h})$ reference depth was greater than the current depth, resulting in a gauge evaporation deficit. This biases total precipitation lower since positive increases in gauge depth must surpass the evaporation deficit to be detected. The wAvg method computes depth change over a smaller $5 \mathrm{~min}$ period, and therefore the magnitude of evaporative losses over the calculation window is smaller than pairwise method. As a result, the wAvg method has a much smaller evaporation deficit compared to pairwise evident from Fig. 8a-f. In event 18, the overestimation of reference depths using the pairwise method resulted in missed precipitation that was captured by the wAvg method. For this same event, precipitation was calculated with the pairwise method having little or no negative depth change (not shown) prior to precipitation from the Geonor-NonEvap (control) gauge, suggesting that QA processes within pairwise were not the cause of the precipitation bias. For the wAvg method, the same total precipitation $(0.3 \mathrm{~mm})$ for event 18 was reported from both Geonor-Evap and Geonor-NonEvap gauges, suggesting this method was less sensitive to gauge evaporation. In addition to under-reporting total precipitation, poor evaluations of reference depths due to gauge evaporation bias caused the pairwise algorithm to report precipitation later in time for event 25 (Fig. 8c and d) and with different subhourly intensities for event 16 (Fig. 8e and f) relative to wAvg. Shifts in the timing and intensity of precipitation can occur when precipitation events start off light (low precipitation rates) and changes in gauge depth over the initial couple of 5 min periods using the pairwise approach are not sufficient to overcome the evaporation deficit and exceed the $0.2 \mathrm{~mm}$ threshold.

\section{Conclusions}

The gauge evaporation field campaign revealed that evaporation from the Geonor T200B all-weather precipitation gauge, used by the USCRN network, was extensive and larger (greater RMSE values) than variations in gauge depth due to sensor noise observed from the Geonor-NonEvap gauge. Hourly evaporation rates from the Geonor-Evap gauge were on average $0.1 \mathrm{~mm}$, but had higher evaporation rates during daylight hours, particularly during the afternoon period. Accumulated evaporative loss over the dry period (no precipitation) from the Geonor-Evap gauge was $228.5 \mathrm{~mm}$ over the 3-month study period, which cannot be explained by sensor noise alone. Over this same time period, the control gauge (Geonor-NonEvap) with an evaporative suppressant lost a fraction of that amount $(2.6 \mathrm{~mm})$ with a distribution of depth changes nearly systemic about zero (small positive and negative depth change). The larger depth variations from the Geonor-Evap gauge suggest that gauge evaporation has a more significant effect on gauge depth measurements than sensor noise.

In line with previous studies, evaporative losses were more pronounced during dry conditions when evaporative demand was greater (dry and warm conditions during the afternoon hours). However, as evaporative demand diminished over the evening hours, sensor noise seemed to become a more dominate source of depth variations from the Geonor-Evap gauge. The change in dominate error sources between evaporation (daylight hours) or sensor noise (nocturnal hours) combined with condensation buildup may help to explain the small positive mean depth change during the early morning hours (05:00 LT) and the larger diurnal range in mean depth change from the Geonor-Evap gauge over dry hours. These results suggest that precipitation gauges sensitive to evaporation can experience greater diurnal oscillations in gauge depth if an insufficient amount of an evaporation suppressant is in the gauge. The larger variations may further challenge QA processes that try to distinguish between noise and precipitation without a collocated instrument (precipitation detector) that can monitor the presence of precipitation.

Gauge evaporation impacted precipitation measurements from the two QA methods differently. The algorithm averaging past gauge data to minimize the effects of wire noise (pairwise) and evaluate depth change had larger differences between the Geonor-Evap and Geonor-NonEvap (control) 

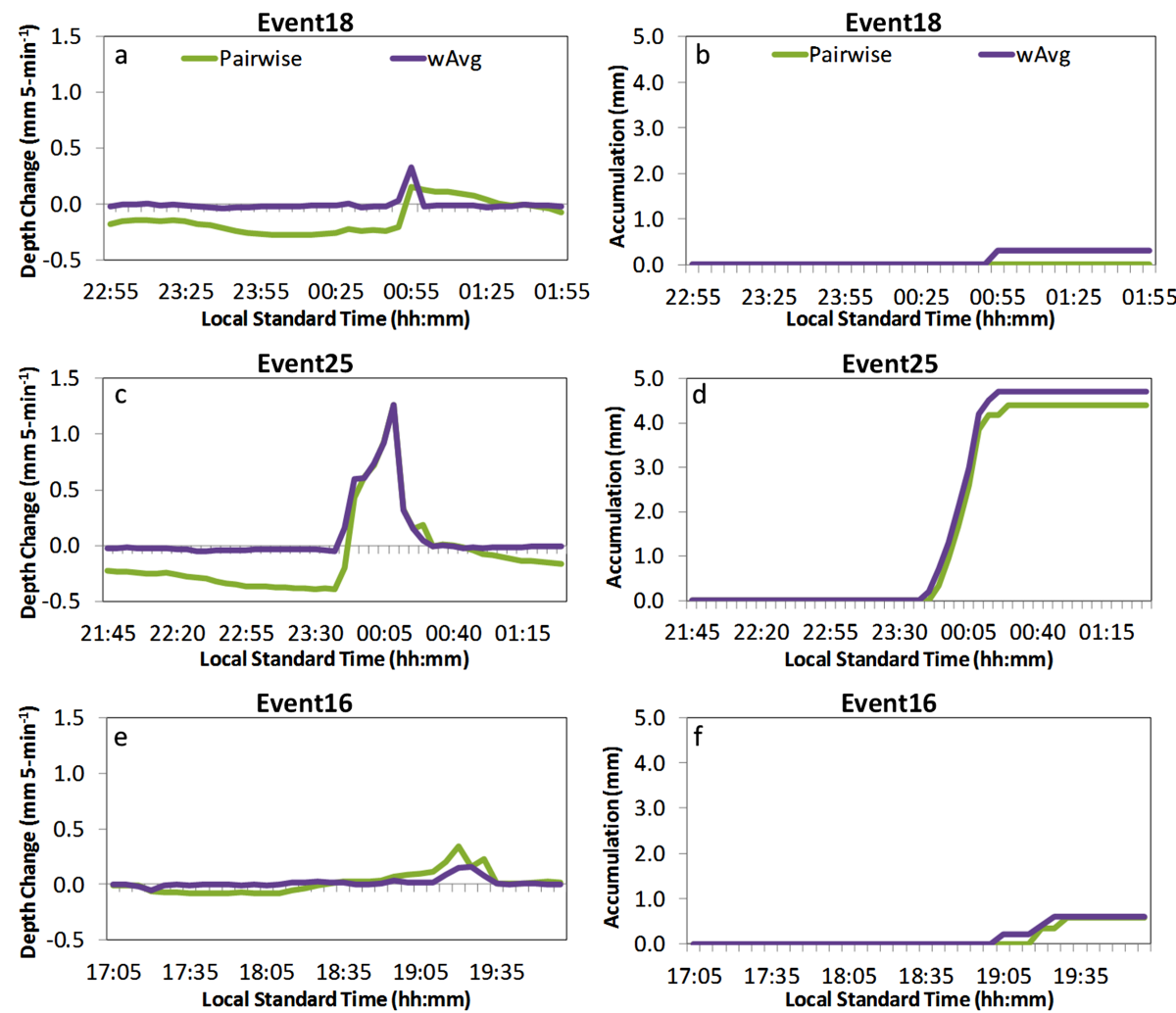

Figure 8. Left: sub-hourly computed depth changes from pairwise and wAvg algorithms and (right) reported sub-hourly precipitation from the Geonor-Evap gauge for precipitation events 18 (a and b), 25 (c and d), and 16 (e and f).

gauges compared to the wAvg approach. This was attributed to the length of the calculation period used to determine depth change. The pairwise approach has a much larger calculation period $(2 \mathrm{~h})$ compared to the wAvg method that calculates depth change over a 5 min period. Moreover, the magnitude of evaporative losses that occur over the calculation period will be larger for the pairwise than wAvg method, increasing its sensitivity to gauge evaporation. The shorter calculation period may help to explain why the wAvg method resulted in much smaller discrepancies in precipitation totals between the Geonor-Evap and Geonor-NonEvap gauges over the study period.

The performance of the wAvg algorithm to account for gauge evaporation in field tests agrees with an earlier study using synthetic precipitation events and simulated gauge evaporation rates (Leeper et al., 2015b). These combined studies demonstrate that the wAvg approach to calculating precipitation is less sensitive to gauge evaporation than the pairwise algorithm and is a more suitable method to monitor USCRN station precipitation. Additionally, these studies also suggest that while gauge design and atmospheric conditions affect gauge evaporation rates, algorithms used to evaluate depth change can influence the magnitude of evaporation biases on precipitation measurements, as suggested by Sevruk et al. (2009). This is important for networks wanting to mon- itor precipitation from protected wilderness areas where the use of evaporative suppressants may be restricted or where the additional maintenance and disposal of the additive is too costly. This may also be of interest to networks that typically use suppressants to ensure that QA methods do not compromise measurement accuracy when an insufficient quantity of a suppressant is in a gauge over the summer months.

However, additional follow-up studies covering longer time spans (over multiple evaporation seasons) from additional locations is necessary to evaluate these results further. In addition, this does not suggest that evaporative suppressants are unnecessary year round. In a network like the USCRN that is designed to measure both solid and liquid precipitation, evaporation suppressants must still be used during the winter to suppress the evaporation of antifreeze chemicals necessary to measure the liquid equivalent of frozen hydrometeors. With that said, this short-term field study demonstrates that the wAvg algorithm limits the effects of gauge evaporation over summer months, improving the accuracy of the USCRN precipitation record. This study will help guide the development and evaluation of precipitation algorithms for weighing bucket gauges that are prone to evaporation, which extends beyond the Geonor gauge and USCRN setup. To foster additional studies and collaboration, the field campaign data presented here are available as an online sup- 
plement for others to evaluate the effects of gauge evaporation on their QA methods.

Acknowledgements. This work was supported by NOAA through the Cooperative Institute for Climate and Satellites - North Carolina under Cooperative Agreement NA09NES4400006 in addition to support for USCRN from the NOAA Climate Program Office. We want to thank Al Jachcik and Mark Hall for the experimental setup, monitoring, and data collection as well as Michael Palecki and Scott Embler for their comments on USCRN precipitation system. A special thanks to reviewers for their editorial comments. The views and opinions, and findings contained in this report are those of the authors and should not be construed as an official NOAA or US Government position, policy or decision.

Edited by: D. Yang

\section{References}

Aaltonen, A., Elomaa, E., Touminen, A., and Valkovuori, P.: Measurement of Precipitation. Proc. Symp. On Precipitation and Evaporation, Vol. 1, Slovak Hydrometeorical Institute and Swiss Federal Institute of Technology, Bratislava, Slovakia, 42-46, 1993.

Baker, B. C., Buckner, R., and Collins, W.: Calculation of USCRN precipitation from Geonor weighing precipitation gauge, NOAA Technical Note, Rep. USCRN-05-1, NOAA, Asheville, USA, 2005.

Duchon C. E.: Using vibrating-wire technology for precipitation measurements, in: Precipitation Advances in Measurement, Estimation and Prediction, edited by: Michaelides, S. C., Springer, Germany, 33-58, 2008.

Duchon, C. E. and Essenberg, G. R.: Comparative rainfall observations from pit and aboveground rain gauges with and without wind shields, Water Resour. Res., 37, 3253-3263, 2001.

Dunne, T. and Leopold, L. B.: Water loss from lakes, in: Water in Environmental Planning, W. H. Freemand and Company, San Francisco, USA, 95-125, 1978.

Golubev, V. S., Groisman, P. Y., and Quayle, R. G.: An Evaluation of the United States Standard 8-in. nonrecording raingauge at the Valdai Ploygon, Russia, J. Atmos. Ocean. Tech., 9, 624-629, 1992.

Goodison, B. E., Ferguson, H. L., and McKay, G. A.: Comparison of point snowfall measurement Techniques, Handbook of Snow, edited by: Gray, D. M., and Male, M. D., Pergamon Press, Canada, 200-210, 1981.

Lanza, L., Leroy, M., van der Meulen, J., and Ondras, M.: The WMO laboratory intercomparison of rainfall intensity gauges, Instruments and observing methods, World Meteorol. Org., Geneva, Switzerland, 8 pp., 2005.
Leeper, R. D., Rennie, J., and Palecki, M. A.: Observational Perspectives from U.S. Climate Reference Network (USCRN) and Cooperative Observer Program (COOP): Temperature and Precipitation Comparison, J. Atmos. Ocean. Tech., 32, 703-721, doi:10.1175/JTECH-D-14-00172.1, 2015a.

Leeper R. D., Palecki, M. A., and Davis, J.: Methods to calculate precipitation from weighting bucket gauges with redundant depth measurements, J. Atmos. Ocean. Tech., doi:10.1175/JTECH-D14-00185.1, in press, $2015 b$.

Rasmussen, R., Baker, B., Kochendorfer, J., Meyers, T, Landolt, S., Fischer, A. P., Black, J., Thériault, J.M., Kucera, P., Gochis, D., Smith, C., Nitu, R., Hall, M., Ikeda, K., and Gutmann, E.: How Well Are We Measuring Snow: The NOAA/FAA/NCAR Winter Precipitation Test Bed, B. Am. Meteorol. Soc., 93, 811829, 2012.

Sevruk, B.: Evaporation losses from containers of Hellman precipitation gauges, Hydrol. Sci. Bull., 19, 231-236, doi:10.1080/02626667409493901, 1974.

Sevruk, B. and Hamon, W. R.: International comparison of national precipitation gages with a reference pit gage, World Meteorol. Org., Geneva, Switzerland, 17, 111 pp., 1984.

Sevruk, B., Ondrás, M., and Chvíla, B.: The WMO precipitation measurement intercomparisons, Atmos Res., 92, 376-380, 2009.

Sieck, L. C., Burges, S. J., and Steiner, M.: Challenges in obtaining reliable measurements of point Rainfall, Water Resour. Res., 43, 1-23, doi:10.1029/2005WR004519, 2007.

Strangeways, I.: Improving precipitation measurements, Int. J. Climatol., 24, 1443-1460, doi:10.1002/joc.1075, 2004.

Walsh, J., Wuebbles, D., Hayhoe, K., Kossin, J., Kunkel, K., Stephens, G., Thorne, P., Vose, R., Wehner, M., Willis, J., Anderson, D., Doney, S., Feely, R., Hennon, P., Kharin, V., Knutson, T., Landerer, F., Lenton, T., Kennedy, J., and Somerville, R.: Our Changing Climate, in: Climate Change Impacts in the United States: The Third National Climate Assessment, edited by: Melillo J. M., Richmond, T. C., and Yohe, G. W., U.S. Global Change Research Program, 19-67. doi:10.7930/J0KW5CXT, 2014.

Wang, J. and Wolff, D. B.: Evaluation of TRMM ground-validation radar-rain errors using rain gauge measurements, J. Appl. Meteorol. Clim., 49, 310-324, doi:10.1175/2009JAMC2264.1, 2010.

World Meteorological Organization: Measurement of precipitation, Guide to Meteorological Instruments and Methods of Observation, World Meteorol. Org, Geneva, Switzerland, Rep. 8, 2008.

Yang, D., Goodison, B. E., Metcalfe, J. R., Golubev, V. S., Bates, R., Pangburn, T., and Hanson, C. L.: Accuracy of $8^{\prime \prime}$ standard nonrecording precipitation gauge: results and application of WMO intercomparison, J. Atmos. Ocean. Tech., 15, 54-68, 1998. 